\title{
Neurofeedback training in children with ADHD: 6-month follow-up of a randomised controlled trial
}

\author{
Holger Gevensleben • Birgit Holl • Björn Albrecht • Dieter Schlamp • Oliver Kratz • \\ Petra Studer • Aribert Rothenberger · Gunther H. Moll • Hartmut Heinrich
}

Received: 8 September 2009/Accepted: 31 March 2010/Published online: 25 May 2010

(c) Springer-Verlag 2010

\begin{abstract}
Neurofeedback (NF) could help to improve attentional and self-management capabilities in children with attention-deficit/hyperactivity disorder (ADHD). In a randomised controlled trial, NF training was found to be superior to a computerised attention skills training (AST) (Gevensleben et al. in J Child Psychol Psychiatry 50(7): 780-789, 2009). In the present paper, treatment effects at 6-month follow-up were studied. 94 children with ADHD, aged 8-12 years, completed either 36 sessions of NF training $(n=59)$ or a computerised AST $(n=35)$. Pretraining, post-training and follow-up assessment encompassed several behaviour rating scales (e.g., the German ADHD rating scale, FBB-HKS) completed by parents. Follow-up information was analysed in 61 children (ca. $65 \%$ ) on a per-protocol basis. 17 children (of 33 dropouts) had started a medication after the end of the training or early in the follow-up period. Improvements in the NF group $(n=38)$ at follow-up were superior to those of the
\end{abstract}

Trial registry: ISRCTN87071503. Comparison of neurofeedback and computerised attention skills training in children with attention-deficit/ hyperactivity disorder (ADHD; http://www.controlled-trials.com/ ISRCTN87071503).

H. Gevensleben · B. Albrecht · A. Rothenberger

Child and Adolescent Psychiatry, University of Göttingen,

v.Siebold-Str. 5, 37075 Göttingen, Germany

O. Kratz · P. Studer · G. H. Moll · H. Heinrich

Department of Child and Adolescent Mental Health,

University of Erlangen-Nürnberg, Schwabachanlage 6+10,

91054 Erlangen, Germany

B. Holl · D. Schlamp · H. Heinrich $(\bowtie)$

Heckscher-Klinikum, Deisenhofener Straße 28,

81539 Munich, Germany

e-mail: hheinri@arcor.de control group $(n=23)$ and comparable to the effects at the end of the training. For the FBB-HKS total score (primary outcome measure), a medium effect size of 0.71 was obtained at follow-up. A reduction of at least $25 \%$ in the primary outcome measure (responder criterion) was observed in $50 \%$ of the children in the NF group. In conclusion, behavioural improvements induced by NF training in children with ADHD were maintained at a 6-month follow-up. Though treatment effects appear to be limited, the results confirm the notion that NF is a clinically efficacious module in the treatment of children with ADHD.

Keywords ADHD - Neurofeedback ·

Randomised controlled trial (RCT) · Follow-up ·

Children

\section{Introduction}

For attention-deficit/hyperactivity disorder (ADHD), European guidelines recommend a multimodal treatment tailored to the requirements of the child [29]. Medication (first-line treatment: methylphenidate), cognitive-behaviour therapy and parental training have proven to be effective [22, 31]. But there is still a need for further effective treatment strategies in improving attentional and self-management capabilities in children with ADHD, especially concerning long-term effects [20, 23]. Recent neurofeedback (NF) studies obtained encouraging results and raise the hope of closing the gap in providing children strategies for better self-regulation and -management [9, $12,28]$.

$\mathrm{NF}$ aims at acquiring self-control over certain brain activity patterns, deriving self-regulation strategies, and implementing these self-regulation skills in daily life. 
Two training protocols - theta/beta training and training of slow cortical potentials (SCPs)_are typically used for children with ADHD [15].

In theta/beta training, children learn to reduce activity in the theta band of the EEG $(4-8 \mathrm{~Hz})$ and to increase activity in the beta band (13-20 Hz). In the resting EEG, increased slow wave (theta) activity and/or reduced relative alpha (8$13 \mathrm{~Hz}$ ) and beta activity was reported in several studies on children with ADHD (for review, see [2, 3]). Thus, theta/ beta training may address an underlying neuronal dysfunction. On the other hand, NF may simply be seen as a tool for enhancing specific cognitive or attentional states (an alert and focused but relaxed state in theta/beta training), irrespective of supposed neurophysiological deviations [15].

SCPs are changes of cortical electrical activity lasting from several hundred milliseconds to several seconds. They are thought to represent task-dependent short-term mobilisations of cortical processing resources. While negative SCPs reflect increased excitation (e.g., during states of behavioural or cognitive preparation), positive SCPs indicate reduction of cortical excitation of the underlying neural networks (e.g., during behavioural inhibition) [4].

The contingent negative variation (CNV) is an SCP that reflects anticipation and/or preparation [18]. It is, for example, elicited in cue trials of a continuous performance test. In event-related potential studies, the CNV was found to be reduced in children with ADHD (for review, see [2]). Training of SCPs leads to an increase of the CNV [14]. Thus, SCP training, in which surface-negative and surfacepositive SCPs have to be generated over the sensorimotor cortex, could help children with ADHD to improve their assumed dysfunctional regulation of energetical resources [26].

In the last decade, several NF studies in children with ADHD have been published which manage to overcome the methodological shortcomings of earlier studies [9, 10, $14,21,28]$. In all of these studies, positive behavioural, cognitive and/or neurophysiological effects were described. Our group conducted a randomised controlled trial encompassing 102 children with ADHD. In this trial, behavioural and neurophysiological effects of NF, which included one training block of theta/beta training and one block of SCP training, were analysed in comparison to a computerised attention skills training (AST) [12, 13, 32]. According to parent and teacher ratings, children of the NF group showed larger behavioural improvements than those of the control group (medium effect size of 0.6 for the primary outcome measure, total score of the German ADHD rating scale, FBB-HKS [7]). Due to comparable settings and demands for NF and the control training, superiority of NF was first and foremost ascribed to specific factors. A tendency for larger improvements was observed if theta/beta training preceded SCP training [12].

At the neurophysiological level (resting EEG, eventrelated potentials), specific associations with behavioural improvements could be revealed for theta/beta and SCP training (e.g., association between decrease of theta activity and reduction of ADHD symptomatology) [13, 32]. These neurophysiological effects contribute to a better understanding of the mechanisms underlying a successful training and indicate specificity of NF training effects.

Both behavioural and neurophysiological findings of our trial indicate that NF may be considered as a clinically efficacious module in the treatment of children with ADHD.

One of the questions which has not been studied under controlled conditions is whether NF training effects remain stable after completing the training. Leins et al. [17] reported that children with ADHD, who had participated in either a theta/beta training or an SCP training, were able to learn cortical self-regulation accompanied by significant improvements in behaviour and cognition. These effects remained constant after 6 months. For a subgroup of 23 (from initially 47) children, 2-year follow-up data could also be assessed [11]. Neuroregulation skills were still preserved. Behavioural and cognitive effects were reported to be stable or even further enhanced. However, due to the lack of a control group, the effects cannot be differentiated from the natural course.

This paper reports follow-up behavioural data assessed 6 months after completion of the training (either NF training or AST) for the children with ADHD of our previous paper [12]. We hypothesised that behavioural improvements in the NF group remain stable and superior to those of the control group.

\section{Materials and methods}

\section{Subjects}

102 children with ADHD (8-12 years) participated in a NF training or an AST. Subjects were randomly assigned to one of the two study groups (ratio NF, control training $=3: 2$; see also Fig. 2). Eight children (NF, $n=5$; AST, $n=3$ ) discontinued the study due to immediate need for medical treatment $(n=3)$, organisational problems of the parents $(n=2)$, loss of motivation $(n=1)$ or protocol violation $(n=2)$. Sample size had been estimated a priori to be large enough to detect a medium effect size of about 0.5 with a power of 0.8 (one-sided, 0.05 -level test).

Table 1 summarises inter alia demographic, psychological and clinical variables of the children completing their training. Concerning these variables, there were no 


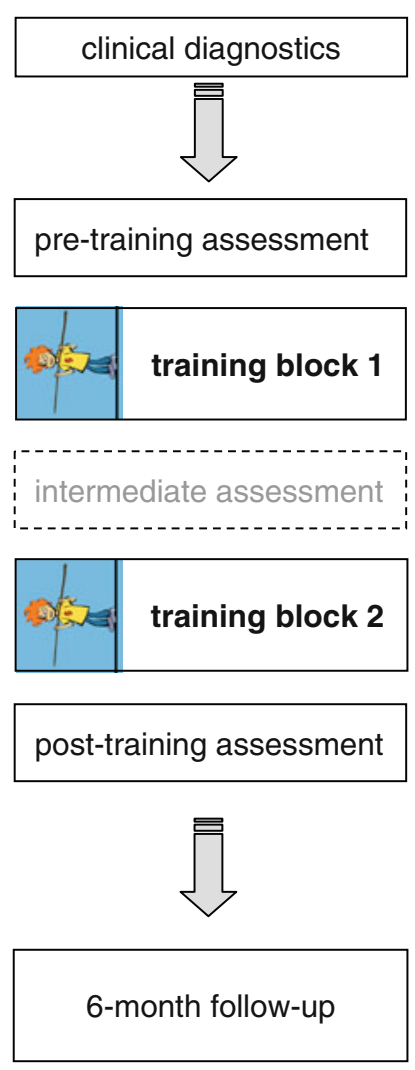

Fig. 1 Schematic illustration of the design of the randomised trial in children with ADHD. The training (neurofeedback, NF; attention skills training) was divided into two blocks. Children of the NF group conducted theta/beta training in one block and SCP training in the other block (balanced order). Behavioural ratings used for follow-up evaluation were assessed before the training started, directly after the end of the training and 6 months after the end of the training

significant differences between NF group $(n=59)$ and control group $(n=35){ }^{1}$

All patients fulfilled DSM-IV criteria for ADHD [1]. Diagnoses were based on a semi-structured clinical interview (CASCAP-D [6]) and confirmed using the Diagnostic Checklist for Hyperkinetic Disorders/ADHD [7] by a child and adolescent psychiatrist or a clinical psychologist, supervised by a board-certified child and adolescent psychiatrist. With mean total FBB-HKS scores of about 1.5 (range 0-3; see Table 2), ADHD symptomatology was moderately pronounced in both training groups. Children with comorbid disorders other than conduct disorder, emotional disorders, tic disorder and dyslexia were excluded from the study. All children lacked gross neurological or other organic disorders. All children were drug-free for at least 6 weeks before starting the training and without concurring psychotherapy.

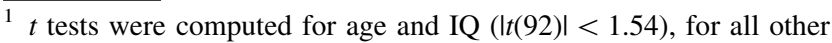
variables $\chi^{2}$ tests were applied $\left(\chi^{2}<2.19\right)$.
}

The study follows the CONSORT guidelines for randomised trials [5]. It was approved by the local ethics committees of the participating clinics and conducted according to the declaration of Helsinki. Assent was obtained from the children and written informed consent from their parents.

\section{Design of the study}

The design of the study is illustrated in Fig. 1. NF and AST both consisted of 36 units of 50 min each. Both treatments were divided in two blocks of 18 units. These 18 units were combined in 9 sessions. These sessions took place two to three times a week. The NF training consisted of 1 block of 18 units of theta/beta training and 1 block of 18 units of SCP training (balanced order). For both NF and AST training, there was a break of 2-3 weeks between the two treatment blocks. The NF and the AST training were designed as similarly as possible concerning the setting and the demands upon the participants, e.g., both treatments encompassed attention demanding tasks on a computer (to a comparable amount, 25-30 min per training unit), acquirement of strategies for focussing attention, and efforts to transfer learned strategies into daily life [12]. Parents were not explicitly informed about the treatment condition of their child (NF vs. AST). ${ }^{2}$

Parent ratings were assessed in the week before the training course started (pre-training), about 1 week after the last session of the first (intermediate ${ }^{3}$ ) and second training block (post-training), respectively, and 6 months after the end of the training (follow-up).

In contrast to the other assessment points, neuropsychological/physiological data were not measured at follow-up.

\section{Training programs}

\section{Neurofeedback}

The NF system SAM ("self-regulation and attention management") which was developed by our study group was used for NF training.

In theta/beta training, the task was to reduce theta and enhance beta activity. A bar on the left of the screen (representing theta activity) had to be reduced while simultaneously a bar on the right (representing beta

\footnotetext{
2 At the post-training assessment, about $40 \%$ of the parents could not reliably quote treatment assignment of their child. The attitude of the parents in the two groups towards the treatment of their child and post hoc evaluation of the training did not differ (rated via "placebo scales").

3 These ratings will not be considered for the evaluation of follow-up results in this paper.
} 
Table 1 Demographic and clinical characteristics of the NF group and the control group

\begin{tabular}{|c|c|c|c|c|}
\hline & \multicolumn{2}{|l|}{ NF group } & \multicolumn{2}{|l|}{ Control group } \\
\hline & $\begin{array}{l}\text { Post-training sample } \\
(n=59)\end{array}$ & $\begin{array}{l}\text { Follow-up sample } \\
(n=38)\end{array}$ & $\begin{array}{l}\text { Post-training sample } \\
(n=35)\end{array}$ & $\begin{array}{l}\text { Follow-up sample } \\
(n=23)\end{array}$ \\
\hline Age (years; month) & $9 ; 10 \pm 1 ; 3$ & $9 ; 11 \pm 1 ; 4$ & $9 ; 4 \pm 1 ; 2$ & $9 ; 5 \pm 1 ; 1$ \\
\hline Sex (boys/girls) & $51 / 8(86.4 \% / 13.6 \%)$ & $32 / 6(84.2 \% / 15.8 \%)$ & $26 / 9(74.3 \% / 25.7 \%)$ & $16 / 7(69.6 \% / 30.4 \%)$ \\
\hline IQ (HAWIK-III [30]) & $106.1 \pm 13.2$ & $106.5 \pm 13.3$ & $104.5 \pm 12.9$ & $106.8 \pm 13.0$ \\
\hline \multicolumn{5}{|l|}{ DSM-IV subtype } \\
\hline Combined type & $39(66.1 \%)$ & $23(60.5 \%)$ & $27(77.1 \%)$ & $17(73.9 \%)$ \\
\hline Inattentive type & $20(33.9 \%)$ & $15(40.5 \%)$ & $8(22.9 \%)$ & $6(26.1 \%)$ \\
\hline Drug-naive & $54(91.5 \%)$ & $36(94.7 \%)$ & $33(97.1 \%)$ & $22(95.7 \%)$ \\
\hline \multicolumn{5}{|l|}{ Associated disorders } \\
\hline Conduct disorder & $10(16.9 \%)$ & $5(13.2 \%)$ & $7(20.0 \%)$ & $3(13.0 \%)$ \\
\hline Emotional disorder & $3(5.1 \%)$ & $2(5.2 \%)$ & $3(8.6 \%)$ & $1(4.3 \%)$ \\
\hline Tic disorder & $3(5.1 \%)$ & $1(2.6 \%)$ & $0(0.0 \%)$ & $0(0.0 \%)$ \\
\hline Dyslexia & $12(20.3 \%)$ & $8(21.1 \%)$ & $10(28.6 \%)$ & $6(26.1 \%)$ \\
\hline
\end{tabular}

Data are presented for the children who had completed their training ("post-training sample") and, separately, for the children for whom follow-up data were available ("follow-up sample"). At the pre-training level, there were no significant differences between the groups (neither for the post-training sample nor for the follow-up sample)

activity) had to be increased. In each unit, about five or six trials of $5 \mathrm{~min}$ each, or up to three trials of $10 \mathrm{~min}$ each, were performed. Baseline values of theta and beta activity were determined at the beginning of each session $(3 \mathrm{~min})$. Children were instructed to reach a relaxed but attentive state and to find individual strategies to control the bars.

In SCP training, the children had to generate negative or positive SCPs. They had to find appropriate strategies to direct a ball upwards (negativity trials) or downwards (positivity trials). Negativity (50\%) and positivity trials (50\%) were presented in random order. A trial lasted for $8 \mathrm{~s}$ (baseline period: $2 \mathrm{~s}$, feedback period: $6 \mathrm{~s}$ ). Children were instructed to reach an attentive (negativity trials) or relaxed state (positivity trials). In each SCP training unit, approximately 120 trials were performed, divided into $2-3$ blocks of 40-60 trials in each treatment unit.

For both NF protocols, feedback was calculated from $\mathrm{Cz}$ (reference: mastoids, bandwidth: $1-30 \mathrm{~Hz}$ for theta/beta training and $0.01-30 \mathrm{~Hz}$ for SCP training, respectively, sampling rate: $250 \mathrm{~Hz}$ ). Vertical eye movements, which were recorded with electrodes above and below the left eye, were corrected online using slightly different regression-based algorithms for theta/beta training [25] and SCP training [16]. For segments containing artefacts exceeding $\pm 100 \mu \mathrm{V}$ in the EEG channel and $\pm 200 \mu \mathrm{V}$ in the EOG channel, no feedback was calculated.

Transfer trials, i.e., trials without contingent feedback, were conducted about one-third at the beginning of a training block and about two-third at the end of a training block. These transfer trials, as well as homework, were intended to improve generalisation of treatment effects.

\section{Attention skills training}

The AST was based on "Skillies" (Auer-Verlag, Donauwörth, Germany), a German learning software programme which primarily exercises visual and auditory perception, vigilance, sustained attention, and reactivity. In "Skillies", the children had to sail to several islands. On each island, a clearly defined task-each requiring different attentionbased skills-had to be solved (for further information, see [12]). The training was complemented by some selfdirected interventions from cognitive therapy to assure comparability to NF, i.e., the children were to compile (meta-)cognitive strategies such as focusing attention, careful processing of tasks and impulse control. Corresponding to the NF group, children of the AST group should practice their compiled strategies in daily life situations.

Behavioural assessment

The following questionnaires (assessed at pre-training, post-training and follow-up) were completed by parents to evaluate the follow-up results:

- German ADHD rating scale (FBB-HKS) [7]: The FBB-HKS is a 20-item questionnaire related to DSM-IV and ICD-10 criteria for ADHD and hyperkinetic disorders, frequently used in Germany in the evaluation of medical and cognitive behavioural treatment of ADHD (e.g. [27]). The severity of each item is rated from 0 to 3. Outcome measures were the main FBBHKS total score, i.e., the mean value of all items as well 
Table 2 Parent behaviour ratings (mean values \pm standard deviation) assessed at pre-training, post-training and follow-up for the children with ADHD for whom follow-up data were available ("follow-up sample")

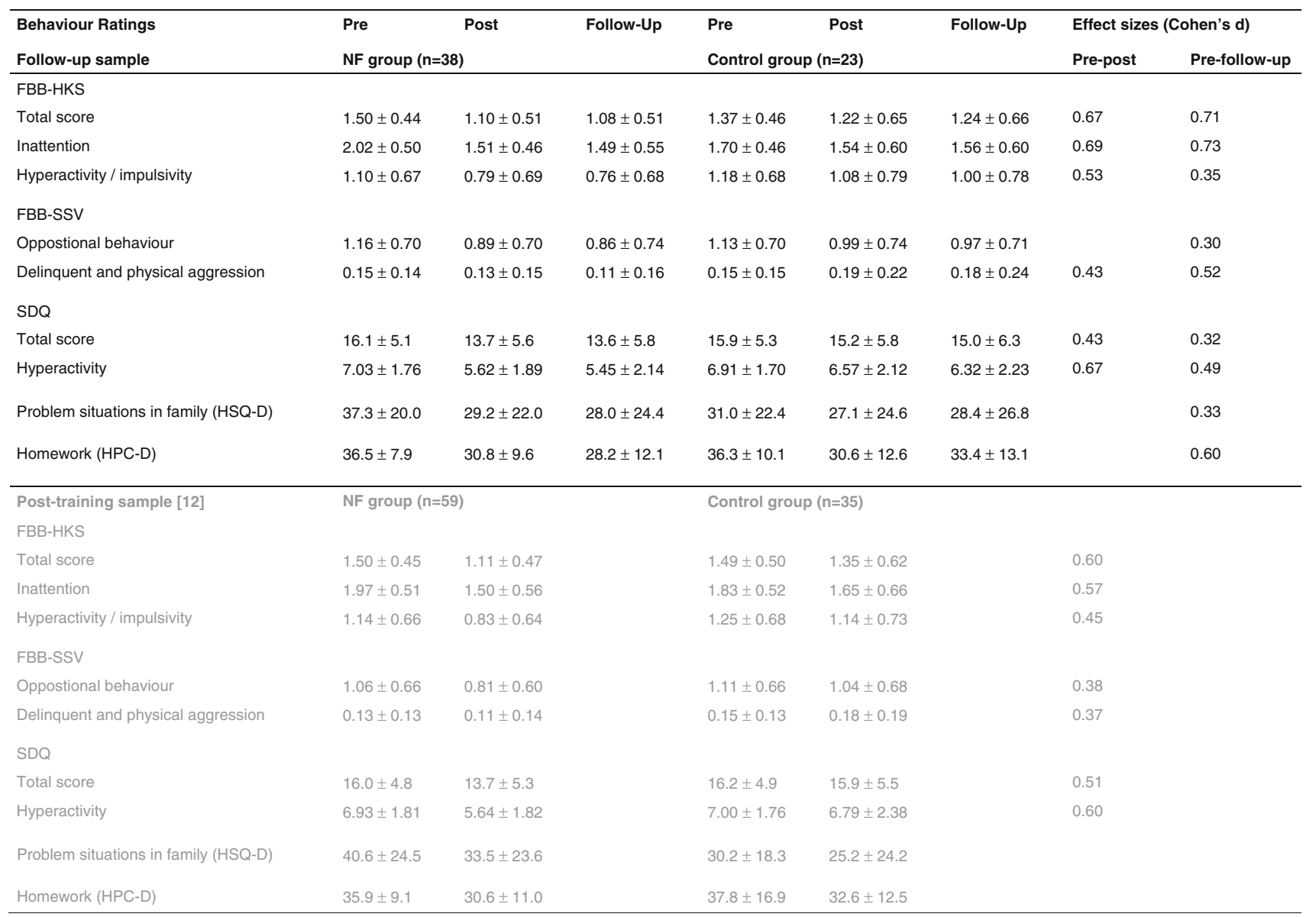

Only effect sizes (Cohen's $d$ ) $\geq 0.3$ are reported. All effect sizes refer to comparisons of the change scores (from pre- to post-training or from pre-training to follow-up) between the training groups. For comparison purposes, the pre-training and post-training measures of all children who had completed the training ("post-training sample") are shown in the lower part (already reported in [12])

as subscores for inattention and hyperactivity/impulsivity. The FBB-HKS total score constituted the primary outcome measure of the study.

- German Rating Scale for Oppositional Defiant/Conduct Disorders (FBB-SSV) [7]: It is comprised of 25 items. The severity of each item is rated from 0 to 3 . Outcome measures were the subscales oppositional behaviour (mean value of the first 9 items) and delinquent and physical aggression (mean value of the remaining 16 items).

- The Strength and Difficulties Questionnaire (SDQ, German version) [24, 33] is comprised of 25 items which address both positive and negative attributes. Each item is rated from 0 to 2 . Outcome measures were the total difficulties score as well as the five subscales (emotional symptoms, conduct problems, hyperactivity, peer problems, prosocial behaviour).
- The Home Situations Questionnaire (HSQ, German version) [8] was used to assess behaviour problems of the child in specific home situations. The HSQ consists of 16 situations in which problematic child behaviour can occur. Parents rate whether the problem behaviour is present in that setting; if so, they rate its severity on a nine-point scale.

- Problem behaviour during homework was assessed using the Homework Problem Checklist (HPC, German version) [8]. This checklist consists of 20 items, rated on a four-point frequency scale.

Data analysis

Per-protocol analysis was conducted to avoid confounding the treatment effects with additional treatment. Children 
were classified as dropouts and excluded if they had started another treatment (e.g., medication, psychotherapy) or if questionnaires were not returned.

Behavioural data were analysed in repeated-measures ANOVAs with between-subject factor GROUP (NF vs. control training), within-subject factor TIME (post-training, follow-up) using the baseline (pre-training) measure as a covariate. If NF training effects are still superior to the control training at follow-up, the ANOVA is expected to reveal a significant GROUP effect. If the difference between the two trainings becomes significantly greater or smaller, this effect is expected to be indicated by a significant GROUP $\times$ TIME interaction.

Effect sizes (Cohen's $d$ ) were calculated as the difference of the change of a measure from pre-training to an assessment point (post-training and follow-up, respectively) and the corresponding change score in the control group divided by the pooled standard deviations of these change scores. To compare the ratio of responders $(\geq 25 \%$ reduction of the primary outcome measure) in the NF group and the control group, the odds ratio was calculated.

Though limited by a smaller sample size, possible effects of the order of the NF training blocks were tested by comparing improvements in the FBB-HKS obtained at post-training and at follow-up ( $t$ tests).

Since there were about 35\% dropouts (see "Results"), we also tested for differences in the clinical (behavioural) data between the dropouts and the remaining children. We computed ANOVAs with between-subject factors GROUP (NF vs. control training) and DROPOUT (dropout vs. follow-up data available) and within-subject factor TIME (pre-training, post-training).

SPSS (v.16) was used for statistical analysis. For all statistical procedures, significance was assumed if $p<0.05$.

\section{Results}

\section{Dropouts}

17 children (NF group: $n=11,18.6 \%{ }^{4}$; control group: $n=6,17.1 \%)$ started a medication during the follow-up interval. None of the children started any other treatment. Parents of 16 children (NF group: $n=10,16.9 \%$; control group: $n=6,17.1 \%$ ) did not return the questionnaires. Thus, there were 33 dropouts with percentages of dropouts not differing between NF and control group $\left(\chi^{2}=0.034\right.$, $d f=2$, n.s.).

\footnotetext{
4 (of these 11) children of the NF group starting a medication had been classified as responders at the end of the training.
}

Analysis of the training effects at 6-month follow-up encompassed 61 of the 94 children completing the training (NF group: $n=38$, control group: $n=23$; see also Fig. 2).

Comparing the dropouts and the remaining children, dropouts were not characterised by significantly smaller training effects, i.e., no significant effect containing the factor TIME (pre- vs. post-training) was obtained in the repeated-measures ANOVAs. Dropouts tended to have higher FBB-HKS scores (FBB-HKS total score: factor DROPOUT: $F(1,90)=3.22 ; p<0.1$; FBB-HKS Inattention subscale: $F(1,90)=3.18 ; p<0.1)$, mainly in the control group as indicated by a trend for the GROUP $\times$ DROPOUT interaction. For all other rating scales, no significant effect or trend containing the factor DROPOUT was obtained.

NF versus control training

Results are summarised in Table 2. Ratings of the children for whom follow-up data were available ("followup sample") are presented in the upper part. To facilitate comparison, the pre-training and post-training measures of all children who completed the treatment ("posttraining sample") are shown in the lower part of Table 2 (already reported in [12]). Only effect sizes $\geq 0.3$ are reported.

For the FBB-HKS total score (primary outcome measure), statistics revealed a significant GROUP effect $(F(1,58)=10.10 ; p<0.005)$ but no significant TIME (post-training vs. follow-up) $(F(1,58)=0.69 ;$ n.s. $)$ or GROUP $\times$ TIME interaction effect $(F(1,58)=0.01 ;$ n.s. $)$. Hence, NF training effects were still superior to the control training at follow-up (medium effect size of 0.71 for change measure from pre-training to follow-up).

Correspondingly, a significant GROUP effect for the FBB-HKS Inattention subscale $(F(1,58)=4.72 ; p<0.05)$ and a trend for the FBB-HKS Hyperactivity/Impulsivity subscale $\quad(F(1,58)=3.45 ; \quad p<0.1) \quad$ were obtained. Reductions of inattention and hyperactivity/impulsivity at follow-up (compared to pre-training) were about 25-30\% in the NF group compared to about $10-15 \%$ in the control group.

Concerning the FBB-SSV subscale Delinquent and Physical Aggression, the ANOVA yielded a trend for the factor GROUP $(F(1,56)=3.64 ; p<0.1)$ due to larger decreases in the NF group. Small to medium effect sizes were obtained at post-training and follow-up, respectively.

For the SDQ subscale Hyperactivity, a significant GROUP effect $(F(1,57)=5.49 ; p<0.05)$ indicated larger improvements for the NF group in comparison to the control group still evident at follow-up. While medium effect sizes were observed for this subscale, small effect sizes resulted for the SDQ total score. For the remaining 
Fig. 2 Patient flow diagram

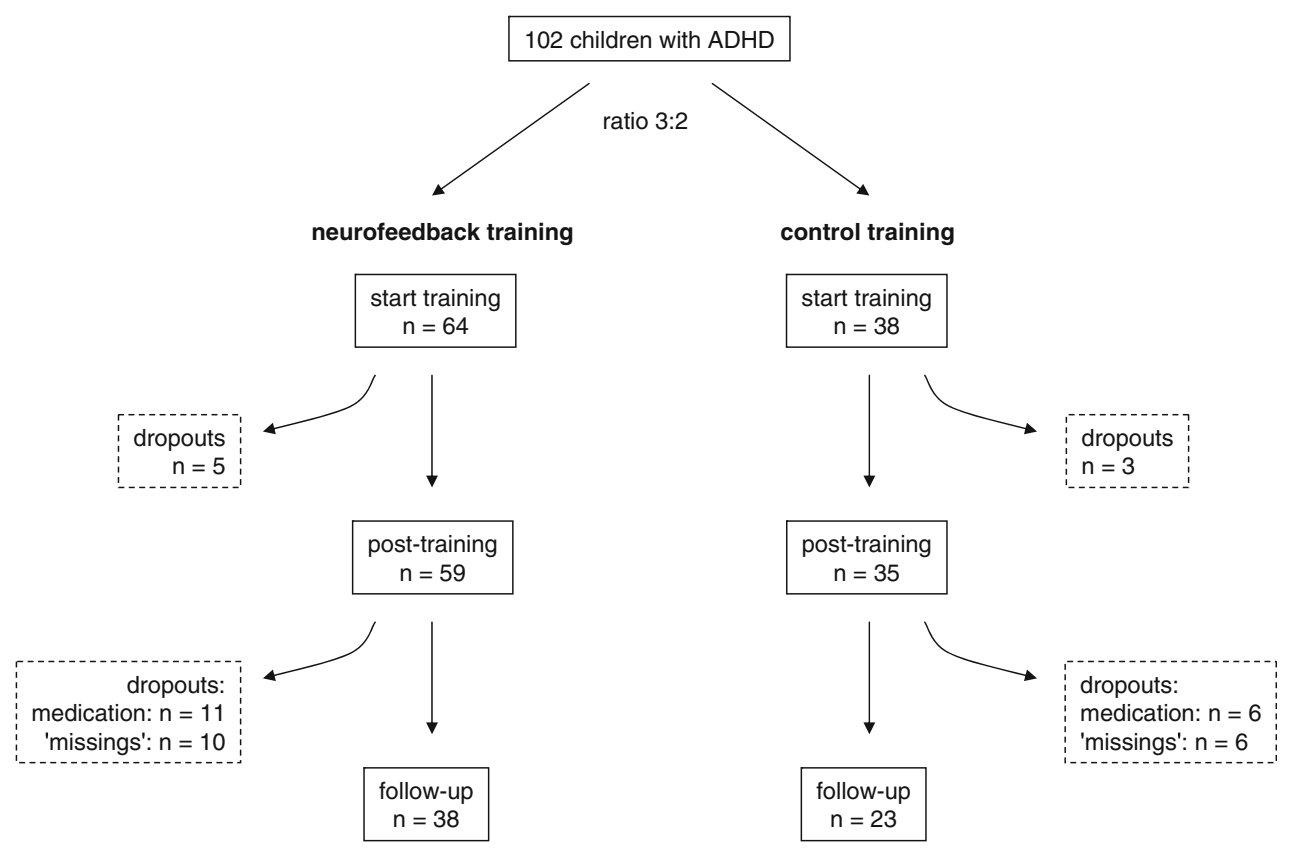

SDQ subscales (not reported in Table 2), effect sizes were smaller than 0.3 .

Concerning homework problems (HPC-D), the GROUP $\times$ TIME interaction turned out to be significant $(F(1,54)=4.18 ; p<0.05)$. Post hoc, we computed an ANOVA with the follow-up score as dependent variable, GROUP as between-subject factor and the pre-training score as covariate. For this analysis, a significant GROUP effect was found $(F(1,54)=5.48 ; p<0.05)$ indicating a larger positive effect concerning homework after NF training (compared to the control training) at follow-up (medium effect size of 0.60). Neither the decrease in NF group $(F(1,32)=1.56$, n.s.) nor the increase in the control group from post-training to follow-up $(F(1,32)=0.02$, n.s.) reached significance.

For the problem situations in family (HSQ-D) questionnaire, no significant effects were obtained.

\section{Responder rate}

In the follow-up sample, 50\% (19 of 38) of the children of the NF group showed a reduction of $25 \%$ or more in the primary outcome measure at post-training and also $50 \%$ at follow-up. According to this criterion, 26.1\% (6 of 23) of the children of the control group were responders at posttraining and $30.4 \%$ ( 7 of 23 ) at follow-up. Odds ratios were 2.83 (post-training) and 2.29 (follow-up) and, thus, in the same range as in the post-training sample (odds ratio: 2.68 [12]) but failed to reach significance due to the smaller sample size (Fisher's exact test, one-sided: post-training: $p=0.06$; follow-up: $p=0.11$ ).
Order of NF protocols

For the follow-up sample, improvements in the FBB-HKS total score at post-training were non-significantly higher when theta/beta training preceded SCP training as compared to the reversed order of protocols $(t(35)=-0.75$, $p=0.46 ; d=0.25)$. The effect size for the post-training sample had been 0.43 [12]. At follow-up, the improvements for both orders were nearly identical $(t(35)=-0.04$, $p=0.97 ; d=0.01$.

\section{Discussion}

The impact of a treatment significantly relies on the generalisation of treatment effects, which can be conceptualised as occurring across settings, behaviour variables and time [19]. This underlines the necessity to consider multiple indicators as well as to assess follow-up measurements concerning the stability of effects. In previous papers, we reported the immediate treatment effects of a NF training (theta/beta training and SCP training) compared to an AST on different outcome levels, encompassing behavioural and neurophysiological measures [12, 13, 32]. This paper deals with the 6-month follow-up analyses of the behavioural outcome. Since some children started medication, we conducted a per-protocol analysis in order to avoid confounding the treatment effect with medication effect. For 61 of the 94 children (ca. 65\%) of this sample, 6-month follow-up data (parent ratings) could be analysed. On average, effects were sustained at follow-up and the 
effects in the NF group were still superior to those of the control group. For the total score of the German ADHD rating scale (primary outcome measure), a medium effect size was obtained. Further, effects were not restricted to core ADHD symptoms but could also be observed in other domains (homework situation, conduct disorder; small to medium effect sizes). Regarding order effects for the NF protocols, the tendency for larger improvements when theta/beta training preceded SCP training could not be confirmed in the follow-up sample.

Since settings and demands for NF and the control training were comparable, these findings indicate that mainly specific effects accounted for the superiority of NF compared to the ASTs.

Specificity of effects is further supported by associations between neurophysiological patterns and the outcome at the clinical (behavioural) level as reported in [13, 32]. For example, in theta/beta training, the decrease of theta activity in the resting EEG was associated with a decrease of the FBB-HKS total score [13]. Concerning SCP training, children with a higher $\mathrm{CNV}$ in an attention test at baseline showed larger improvements after the relatively short training block [32].

On the other hand, partly due to the non-blind design, it cannot be ruled out that unspecific effects might have also contributed to the behavioural effects. ${ }^{5}$

There was a relatively large number of dropouts, i.e., children who either started a medication or for whom no questionnaires were received, at follow-up (about 35\%). However, dropouts were not characterised by a worse training outcome at the end of the training. These children (mainly in the control group) had slightly (but non-significantly) higher scores on the German ADHD rating scale already at the beginning of the training. In general, children for whom follow-up data were available could not be differentiated from dropouts with respect to behavioural or demographic characteristics. So, it seems unlikely that the follow-up results were strongly biased by the large portion of dropouts.

Findings are based on parent ratings only. At the posttraining assessment, for only about $70 \%$ of the children, ratings of the same teacher who had completed the pretraining questionnaires were available. Expecting further high dropout rates due to change of teachers and loss of motivation to complete the questionnaires, we decided not to include teacher ratings in the follow-up analysis. It can be questioned whether teacher ratings would have supported the follow-up results obtained from parent ratings. However, in [17], parent and teacher ratings did not develop differentially from post-training to follow-up. In

\footnotetext{
5 For a more detailed discussion concerning the control condition, see [12].
}

our study, comparable effects resulted for parent and teacher ratings at the post-training assessment [12].

$50 \%$ of the children completing the training were categorised as non-responders, according to a criterion of $25 \%$ reduction in the primary outcome measure. 11 out of 59 children of the NF group started a medication during the follow-up interval. Our study was not designed to achieve maximum NF training effects but had an arguably artificial scientific setting (e.g., separating theta/beta and SCP training in two separate, non-coordinated blocks). Nevertheless, the low responder rate and the portion of children starting a medication in our study argue against NF as a stand-alone intervention for children with ADHD. The results indicate that not every child with ADHD may improve after NF treatment. In our opinion, NF should rather be seen as a treatment module for children with ADHD which can be embedded in a multimodal treatment program tailored to the individual needs of a child.

Stability of training effects at follow-up refers to the mean scores of the NF group and naturally does not apply to all individuals within the group. In our design, we used a fixed number of training sessions. This was intended to standardise number of treatment sessions across all individuals. However, qualitative analyses of our data suggest that children vary in their abilities and speed to learn and apply NF techniques. One block of 18 units for a single protocol might have been too short at least in some children to build up stable regulation capability and to establish transfer into daily life sufficiently. Further research therefore would be required to determine the optimal NF protocol (or combination of protocols) and the adequate number of treatment sessions for a particular child.

Coming back to the long-term outcome, it could be helpful at least for some of the children to conduct further training sessions with longer intervals between the training session to sustain and consolidate regulation capabilities and the transfer into daily life, just as it is usually practiced in conventional cognitive-behaviour therapy. In this respect, the possible benefit of such booster sessions should also be investigated in further studies.

\section{Conclusions}

Behavioural effects of NF training were maintained in children with ADHD at 6-month follow-up, further supporting clinical efficacy of this neurobehavioral training. NF may be recommended as a treatment module for children with ADHD besides conventional behavioural trainings and medication. Future studies should systematically address how to optimise/individualise NF training and how to embed it in a multimodal treatment in children with ADHD. 
Acknowledgments The authors thank Christa Dahlmann, Martin Deinzer, Claudia Vogel, Susanne Wangler, Nicola Wöstmann and the student assistants for their valuable support as well as all the participating families for their contribution and effort. We thank Margaret and Simon Tiffin-Richards for language editing. This study was supported by the German Research Foundation (with a joint grant to H.H., G.H.M, and A.R.; HE 4536/2, MO 726/2, RO 698/4).

\section{References}

1. American Psychiatric Association (1994) Diagnostic and Statistical Manual of Mental Disorders, 4th edition (DSM-IV). American Psychiatric Press, Washington, DC

2. Banaschewski T, Brandeis D (2007) Annotation: what electrical brain activity tells us about brain function that other techniques cannot tell us-a child psychiatric perspective. J Child Psychol Psychiatry 48:415-435

3. Barry RJ, Clarke AR, Johnstone SJ (2003) A review of electrophysiology in attention-deficit/hyperactivity disorder. I. Qualitative and quantitative electroencephalography. Clin Neurophysiol 114:171-183

4. Birbaumer N, Elbert T, Canavan AG, Rockstroh B (1990) Slow potentials of the cerebral cortex and behavior. Physiol Rev 70:1-41

5. Boutron I, Moher D, Douglas G, Altman G, Schulz F, Ravaud P (2008) Extending the CONSORT statement to randomized trials of nonpharmacologic treatment: explanation and elaboration. Ann Intern Med 148:295-310

6. Döpfner M, Berner W, Flechtner H, Lehmkuhl G, Steinhausen HC (1999) Psychopathologisches Befund-System für Kinder und Jugendliche (CASCAP-D). Hogrefe, Göttingen

7. Döpfner M, Lehmkuhl G (2000) DISYPS-KJ-Diagnostik-System für psychische Störungen im Kindes-und Jugendalter. Hans Huber, Bern

8. Döpfner M, Schürmann S, Frölich J (2002) Therapieprogramm für Kinder mit hyperkinetischem und oppositionellem Problemverhalten (THOP). Weinheim, Beltz

9. Drechsler R, Straub M, Doehnert M, Heinrich H, Steinhausen HC, Brandeis D (2007) Controlled evaluation of a neurofeedback training of slow cortical potentials in children with attention-deficit/hyperactivity disorder (ADHD). Behav Brain Funct 3:35

10. Fuchs T, Birbaumer N, Lutzenberger W, Gruzelier JH, Kaiser J (2003) Neurofeedback treatment for attention-deficit/hyperactivity disorder in children: a comparison with methylphenidate. Appl Psychophysiol Biofeedback 28:1-12

11. Gani C, Birbaumer N, Strehl U (2008) Long term effects after feedback of slow cortical potentials and of theta-beta-amplitudes in children with attention-deficit/hyperactivity disorder. Int $\mathrm{J}$ Bioelectromagn 10:209-232

12. Gevensleben H, Holl B, Albrecht B, Vogel C, Schlamp D, Kratz O, Studer P, Rothenberger A, Moll GH, Heinrich H (2009) Is neurofeedback an efficacious treatment for ADHD? A randomised controlled clinical trial. J Child Psychol Psychiatry 50(7):780-789

13. Gevensleben H, Holl B, Albrecht B, Schlamp D, Kratz O, Studer P, Wangler S, Rothenberger A, Moll GH, Heinrich H (2009) Distinct EEG effects related to neurofeedback training in children with ADHD: a randomized controlled trial. Int J Psychophysiol. doi:10.1016/j.ijpsycho.2009.08.005

14. Heinrich H, Gevensleben H, Freisleder FJ, Moll GH, Rothenberger A (2004) Training of slow cortical potentials in attentiondeficit/hyperactivity disorder: evidence for positive behavioral and neurophysiological effects. Biol Psychiatry 55:772-775
15. Heinrich H, Gevensleben H, Strehl U (2007) Annotation: neurofeedback - train your brain to train behaviour. J Child Psychol Psychiatry 48:3-16

16. Kotchoubey B, Schleichert H, Lutzenberger W, Birbaumer N (1997) A new method for self-regulation of slow cortical potentials in a timed paradigm. Appl Psychophysiol Biofeedback 22:77-93

17. Leins U, Hinterberger T, Kaller S, Schober F, Weber C, Strehl U (2006) Neurofeedback for children with ADHD: a comparison of SCP- and theta/beta-protocols. Prax Kinderpsychol Kinderpsychiatr 55:384-407

18. Lütcke H, Gevensleben H, Albrecht B, Frahm J (2008) Brain networks involved in early versus late response anticipation and their relation to conflict processing. J Cogn Neurosci. doi: 10.1162/jocn.2008.21165

19. Mash EJ, Barkley RA (2006) Treatment of childhood disorders, 3rd edn. The Guilford Press, New York

20. Molina BS, Hinshaw SP, Swanson JM, Arnold LE, Vitiello B, Jensen PS, Epstein JN, Hoza B, Hechtman L, Abikoff HB, Elliott GR, Greenhill LL, Newcorn JH, Wells KC, Wigal T, Gibbons RD, Hur K, Houck PR, MTA Cooperative Group (2009) The MTA at 8 years: prospective follow-up of children treated for combined-type ADHD in a multisite study. J Am Acad Child Adolesc Psychiatry 48(5):484-500

21. Monastra V, Monastra D, George S (2002) The effects of stimulant therapy, EEG-biofeedback, and parenting style on the primary symptoms of attention-deficit/hyperactivity disorder. Appl Psychophysiol Biofeedback 27:231-249

22. The MTA Cooperative Group (1999) A 14-month randomized clinical trial of treatment strategies for attention-deficit/hyperactivity disorder. Arch Gen Psychiatry 56:1073-1086

23. Rothenberger A, Döpfner M, Sergeant J, Steinhausen HC (eds) (2004) ADHD beyond core symptoms-not only a European perspective. Eur Child Adolesc Psychiatry, 13 (Suppl 1)

24. Rothenberger A, Becker A, Erhart M, Wille N, Ravens-Sieberer U, the BELLA study group (2009) Psychometric properties of the parent strengths and difficulties questionnaire in the general population of German children and adolescents: results of the BELLA study. Eur Child Adolesc Psychiatry 17(Suppl 1):99-105

25. Semlitsch HV, Anderer P, Schuster P, Presslich O (1986) A solution for reliable and valid reduction of ocular artifacts, applied to the P300 ERP. Psychophysiology 23:695-703

26. Sergeant JA, Oosterlaan J, van der Meere JJ (1999) Information processing and energetic factors in attention-deficit/hyperactivity disorder. In: Quay HC, Hogan A (eds) Handbook of disruptive behavior disorders. Plenum Press, New York, pp 75-104

27. Sevecke K, Döpfner M, Lehmkuhl G (2004) The effectiveness of stimulants of retard forms in children and adolescents with ADHD - a systematic overview. Zeitschrift für Kinder- und Jugendpsychiatrie Psychotherapie 32:265-278

28. Strehl U, Leins U, Goth G, Klinger C, Hinterberger T, Birbaumer N (2006) Self-regulation of slow cortical potentials: a new treatment for children with attention-deficit/hyperactivity disorder. Pediatrics 118:1530-1540

29. Taylor E, Döpfner M, Sergeant J, Asherson P, Banaschewski T, Buitelaar J, Coghill D, Danckaerts M, Rothenberger A, SonugaBarke E, Steinhausen HC, Zuddas A (2004) European clinical guidelines for hyperkinetic disorder-first upgrade. Eur Child Adolesc Psychiatry 13(Suppl 1):I7-I30

30. Tewes U, Rossmann P, Schallberger U (2000) HamburgWechsler-Intelligenztest für Kinder III (HAWIK-III). Hogrefe, Göttingen

31. van der Oord S, Prins PJ, Oosterlaan J, Emmelkamp PM (2008) Efficacy of methylphenidate, psychosocial treatments and their combination in school-aged children with ADHD: a meta-analysis. Clin Psychol Rev 28(5):783-800 
32. Wangler S, Gevensleben H, Albrecht B, Studer P, Rothenberger A, Moll GH, Heinrich H (2010) Neurofeedback in children with ADHD: specific event-related potential findings of a randomized controlled trial (in preparation)
33. Woerner W, Becker A, Rothenberger A (2004) Normative data and scale properties of the German parent SDQ. Eur Child Adolesc Psychiatry 13(Suppl 2):11-16 